\title{
Development of a two-sided relativistic jet in Cygnus X-3
}

\author{
J. Martí1 ${ }^{1}$ J. M. Paredes ${ }^{2}$, and M. Peracaula ${ }^{2}$ \\ 1 Departamento de Física, Escuela Politécnica Superior, Universidad de Jaén, Virgen de la Cabeza 2, 23071 Jaén, \\ Spain \\ 2 Departament d'Astronomia i Meteorologia, Facultat de Física, Universitat de Barcelona, Av. Diagonal 647, \\ 08028 Barcelona, Spain \\ e-mail: josep@am.ub.es, mperacau@am.ub.es
}

Received 4 May 2001 / Accepted 20 June 2001

\begin{abstract}
We present multi-epoch radio maps of the microquasar Cygnus X-3 obtained with the Very Large Array (VLA). The observations were carried out as a Target-of-Opportunity (ToO) project triggered by a series of strong ( $>5$ Jy) radio outbursts in September 2000. We find that Cygnus X-3 was well resolved and clearly elongated in the North-South direction at the VLA resolution in the weeks after the triggering events. In particular, the VLA map from our final epoch of observation perfectly shows the development of a two-sided radio jet extending over $\sim 0.05 \mathrm{pc}$ scales. We interpret both the proper motion and the brightness asymmetry between the jet components in the framework of special relativity effects. Assuming a distance of $10 \mathrm{kpc}$, the true jet velocity that we derive is close to $0.5 c$ with a large angle with the line of sight. The possible connection between the VLA bipolar jets and the elongated one-sided structures observed at milli-arcsecond angular scales is also discussed.
\end{abstract}

Key words. stars: individual: Cyg X-3 - ISM: jets and outflows - X-rays: stars

\section{Introduction}

Cygnus X-3 was originally discovered in X-rays by Giacconi et al. (1967). This X-ray binary has been the subject of intensive study during the last decades, specially after the discovery of its giant radio outbursts in 1972 (Gregory et al. 1972). Today, it is widely accepted as one of the few known microquasar sources in our Galaxy (Mirabel \& Rodríguez 1999). The black hole or neutron star nature of the compact companion in the binary system is still a matter of debate, but a consensus has been reached about the normal companion being a WN WolfRayet star (van Kerkwijk et al. 1996). With this classification, the system belongs to the group of high mass X-ray binaries. The modulation observed in the X-ray and infrared emission every $4.8 \mathrm{~h}$ (e.g. Mason et al. 1986) is interpreted as the orbital period of the system. Thus, the orbit of the compact object around the WN Wolf-Rayet star has to be very tight with semimajor axis of only a few solar radii. Cygnus X-3 has been also proposed to be an emitter of ultra high energy $\gamma$-rays by several groups (see e.g. Murthy \& Wolfendale 1993). The recent discovery of another high mass X-ray binary, LS5039, with a likely $\gamma$-ray counterpart (Paredes et al. 2000) points to microquasars being possibly connected with some galactic $\gamma$-ray sources.

Send offprint requests to: J. Martí, e-mail: jmarti@ujaen.es
Focusing on the Cygnus X-3 flaring radio emission, several authors have provided strong observational evidence indicating that it originates in expanding collimated jet-like structures (see e.g. Martí et al. 2000 and all the references therein). The highest resolution maps of the ejecta have been provided by Mioduszewski et al. (2001) who observed Cygnus X-3 with the Very Long Baseline Array (VLBA) soon after a giant outburst event in 1997. Further support for the jet scenario comes from the agreement between the radio light curves and the predictions from theoretical models of synchrotron emitting radio jets (see e.g. Hjellming \& Johnston 1988; Martí et al. 1992). In spite of this progress, some questions concerning the physical properties of the ejecta remain not yet fully understood: are the jets strongly beamed towards us? What is the true velocity of the flow? Where is the core in the high resolution maps?

The present paper reports new multi-epoch radio images of Cygnus X-3 and its radio jets. Our observations were obtained in $\mathrm{ToO}$ mode, with the triggering event being the recent giant radio outburst in September 2000. Most ToO radio observations of Cygnus X-3 have consisted of mapping the source using Very Long Baseline Interferometry (VLBI) arrays with milli-arcsecond (mas) angular resolution. These VLBI runs are normally carried out as soon as possible after the outburst onset. Contrary to this standard approach, we waited and observed several 
weeks after the onset, with the expectation of studying and imaging the ejecta far away from the central core. The suitable instrument for this project was the VLA of the National Radio Astronomy Observatory (NRAO). Our previous VLA maps of the 1997 outburst, with the central variable core carefully subtracted, indicated that the Cygnus X-3 ejecta is indeed detectable up to one arcsec away from the ejection center (Martí et al. 2000). With these ideas in mind, the main goal of our ToO proposal was to confirm the reality of the extended bipolar radio jets and to obtain a convincing record of their proper motion. The resulting maps have an angular resolution of 0 .' 3 , equivalent to about $0.01 \mathrm{pc}$ at a distance of $10 \mathrm{kpc}$. Since phase calibration is straightforward with the VLA at $\mathrm{cm}$ wavelengths, the maps have no ambiguity in the location of the core, as it happens in some VLBI images. In the following sections, we present the confirmation that Cygnus X-3 is able to develop transient radio jets at arcsecond scales and that they are moving with relativistic speeds. Moreover, the new VLA results open the challenging question of how to relate, consistently, the elongated radio structures seen at very different angular scales.

\section{Observations and data processing}

We observed with the VLA in its most extended A configuration at the $6 \mathrm{~cm}$ wavelength, equivalent to a frequency of $\nu=4.86 \mathrm{GHz}$. Three ToO VLA runs of $5 \mathrm{~h}$ each were scheduled at two-week intervals starting on late October 2000. The data were reduced using the AIPS package of NRAO. The source 3C286 was observed as amplitude calibrator with the range of baselines restricted to $150-300 \mathrm{k} \lambda$ and its nominal flux density reduced by $2 \%$, as recommended in the AIPS CookBook. The phase calibrator used was $2007+404$ at a distance of 4.7 degrees. The log of observations and the bootstrapped flux densities of $2007+404$ are listed in Table 1 .

The observing epochs are indicated in Fig. 1 as vertical arrows overlapped with the $8.3 \mathrm{GHz}$ monitoring provided by the Green Bank Interferometer (GBI). The series of outbursts that triggered the activation of our ToO proposal are clearly visible to the left of the arrows. Consultation of the $15 \mathrm{GHz}$ radio monitoring provided by the Ryle Telescope, kindly posted on the web by G. G. Pooley, indicates that Cygnus X-3 was at a quiescent level of radio emission throughout all the epochs of VLA observation. The vertical dashed line labelled as ejection corresponds to 15 September 2000 (JD 2451802.5), the approximate date when the radio outburst activity started. A very strong outburst event in April 2000 (not shown in Fig. 1) did not trigger our observations because of the inappropriate VLA configuration at that time.

The observation of microquasars with radio interferometers is very often made difficult by the brightness and/or structure changes occurring on time scales of a few hours, i.e., comparable with the typical duration of the observation. The assumption of a constant source, both in brightness and structure, in the commonly used CLEAN
Table 1. Log of VLA observations at $6 \mathrm{~cm}$.

\begin{tabular}{lcc}
\hline Epoch Date & Julian Day $^{\mathrm{a}}$ & $\begin{array}{c}\text { Flux density of } \\
2007+404(\mathrm{Jy})\end{array}$ \\
\hline 21 October 2000 & 2451838.62 & $2.53 \pm 0.02$ \\
$4 / 5$ November 2000 & 2451853.49 & $2.49 \pm 0.02$ \\
$19 / 20$ November 2000 & 2451868.54 & $2.43 \pm 0.01$ \\
\hline
\end{tabular}

a Middle of the observation.

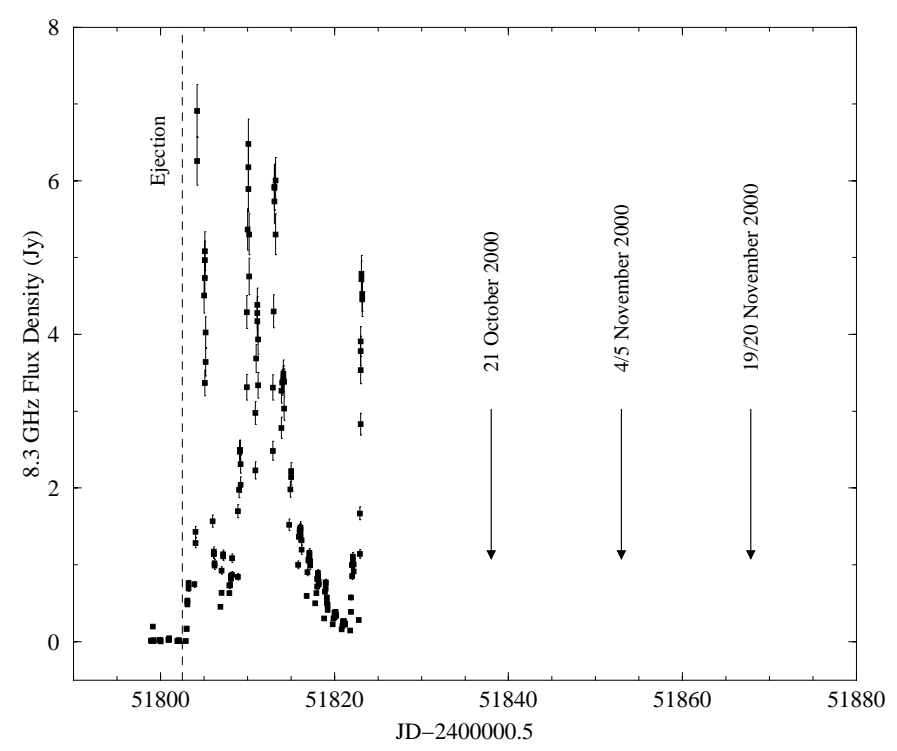

Fig. 1. Radio light curve of Cygnus X-3 as observed at $8.3 \mathrm{GHz}$ by the GBI. A series of strong flaring events are clearly seen reaching up to $\sim 7 \mathrm{Jy}$ at the GBI wavelength. The vertical arrows indicate the three epochs when VLA observations were carried out. The vertical line labelled as ejection corresponds to the starting epoch of the flaring activity that triggered our ToO proposal.

deconvolution algorithm are then clearly violated. At the VLA angular resolution, only the fast radio variability of the Cygnus X-3 core may pose problems for standard data processing within AIPS. Such variability is shown in Fig. 2 and it was especially severe during the first run of our observation. In contrast, the proper motion of the ejecta is not expected to introduce any noticeable changes in the source structure throughout the run duration.

The variability problem in Cygnus X-3 has been addressed by Martí et al. (2000) by subtracting a variable point source with the estimated light curve of the core. When applying this method to their 1997 data, it was possible to clearly see the faint jet-like arcsecond features that otherwise would be masked by the variability artifacts. As it will be seen later, the Cygnus X-3 ejecta in late 2000 were not well detached from the core when we observed with the VLA. Therefore, the Martí et al. (2000) procedure is not straightforward to apply due the difficulties in estimating a good light curve for the core. 

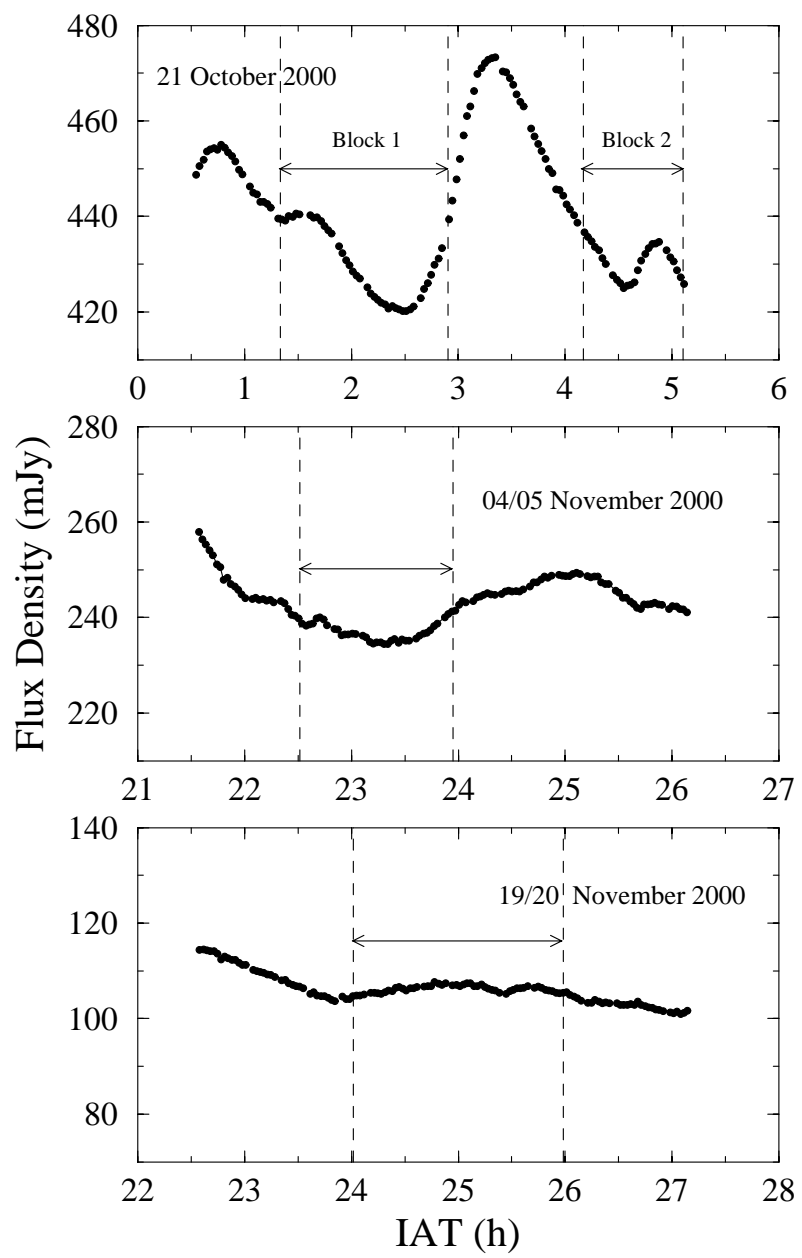

Fig. 2. Radio light curves of Cygnus $X-3$ at the $6 \mathrm{~cm}$ wavelength during the three epochs of VLA observations. Each dot is the average of $2 \mathrm{~min}$ of visibility data using the AIPS task DFTPL. The source was always clearly variable on time scales of hours. The horizontal arrows and vertical lines represent the data block (or blocks) selected to compute the VLA maps in Fig. 6.

Fortunately, Cygnus X-3 behaved in relatively stable way during a significant fraction $(\sim 2 \mathrm{~h})$ of each 2000 observing run. By splitting and using only the visibility data blocks during these intervals, it is possible to compute VLA maps that are practically free of variability problems. The selected intervals are indicated in Fig. 2 by vertical lines. The amplitude of variation during these intervals was always less than $5 \%$. The visibilities were selfcalibrated in phase, using a point source model, prior to performing the splitting.

\section{Results}

In Fig. 3 we show a plot of the amplitude visibility as a function of baseline length on the first epoch 21 October 2000. The decaying trend of the amplitude is the unmistakable signature of a resolved source. Cygnus X-3 was still a very bright radio source $(\sim 0.45 \mathrm{Jy})$ during the first epoch of observation. With such a high flux density, it is

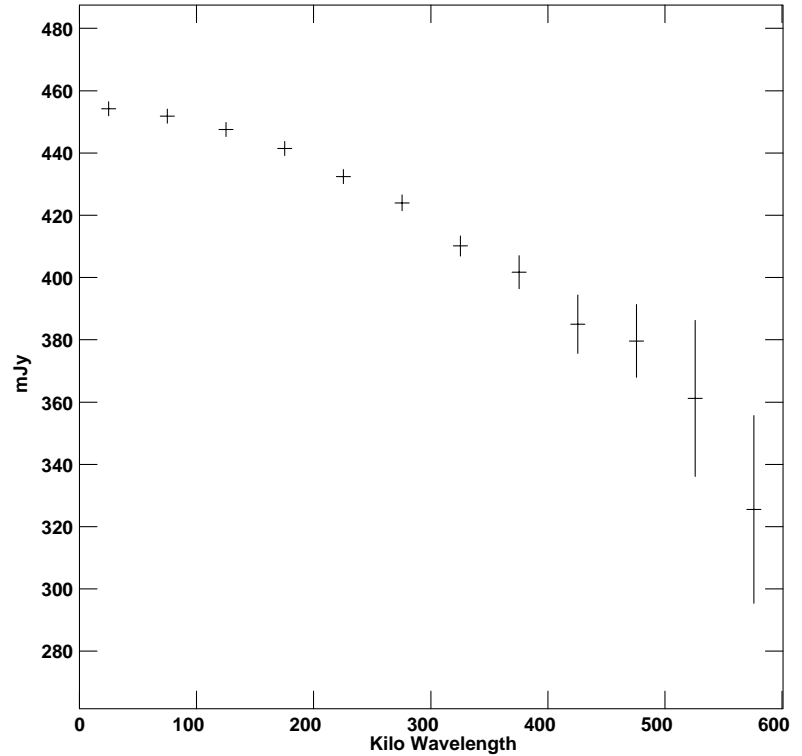

Fig. 3. Plot of the Cygnus X-3 visibility amplitude as a function of the projected baseline on 21 October 2000. The amplitude decay for the longest baselines of the VLA is the clear signature of a resolved source. This plot contains only averaged visibility data at $6 \mathrm{~cm}$ from blocks 1 and 2 of the first epoch.

feasible to carry out reliable model fits in the $u v$ plane every few minutes in order to look for fast structure variations. The AIPS task UVFIT was used for this purpose with an elliptical Gaussian model assumed. The results of the fit, shown in Fig. 4, were fairly stable and clearly indicate an extended elongated radio source. The fact that Cygnus X-3 becomes resolved at the VLA resolution in the weeks following strong radio outbursts is also well evident in Fig. 5. This figure displays the VLA contour map for the third epoch of observation. In this image, the elongated radio source present during the first epoch has produced a remarkable bipolar asymmetric radio jet. For the first time, the jet and counterjet in Cygnus X-3 are clearly detected with no subtraction procedure being necessary to reveal them. The progressive development of radio jets in Cygnus X-3, at arcsecond scales, is better illustrated in Fig. 6. The vertical separation of the different maps in this figure is proportional to the elapsed time between the observing epochs and the direction of the jets has been rotated $90^{\circ}$ counterclockwise.

Assuming that the arcsecond jets were created when the GBI saw the triggering outburst event (JD 2451802.5), the corresponding age of the ejecta is $36.1,51.0$ and 66.0 days for the first, second and third epochs, respectively. For quantitative proper motion estimates, the position of the jet components in the third epoch has been measured by direct model fitting in the $u v$ plane. Two point sources for the jet components plus one elliptical Gaussian for the central core were fitted with UVFIT. In the second epoch, only the northern jet component was fitted since it is the only one visible in the maps. A summary of the fit results for both the core and the jet components 


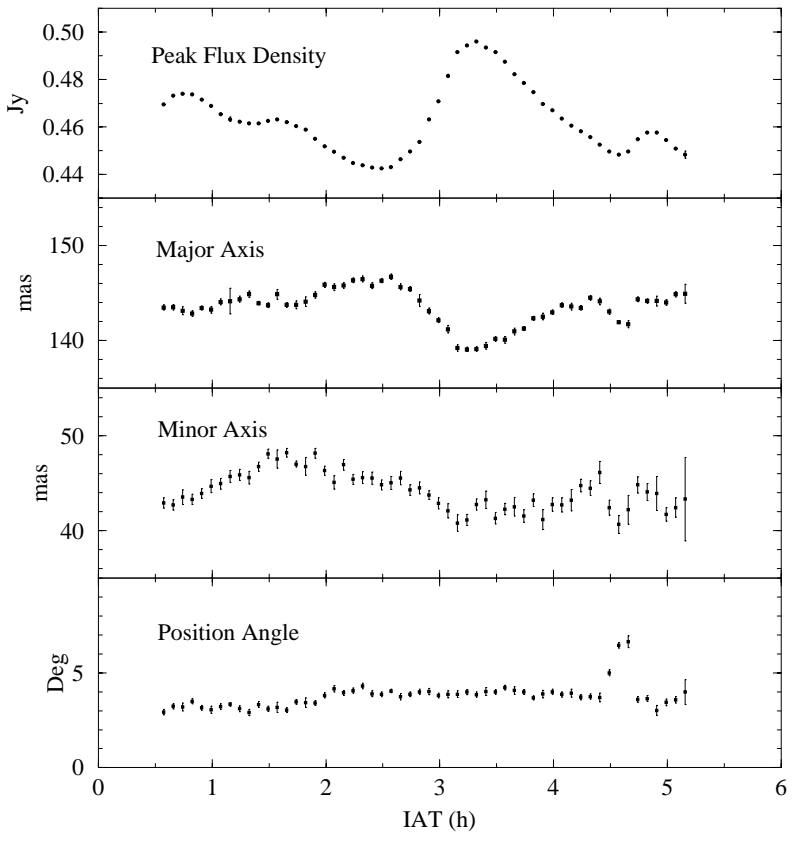

Fig. 4. Best elliptical Gaussian fit to the Cygnus X-3 radio structure on 21 October 2000. The fit was carried out directly in the $u v$ plane using the AIPS task UVFIT at regular intervals of $5 \mathrm{~min}$.

Table 2. Results of an elliptical Gaussian fit in the $u v$ plane for the central core.

\begin{tabular}{|c|c|c|c|}
\hline Epoch & $\begin{array}{c}\text { Angular Size } \\
\left(\operatorname{mas}^{2}\right)\end{array}$ & $\begin{array}{c}\text { Position Angle } \\
\left({ }^{\circ}\right)\end{array}$ & $\begin{array}{c}\text { Flux Density } \\
(\mathrm{mJy})\end{array}$ \\
\hline 1 st & $\begin{array}{c}145.3 \times 45.7 \\
\pm 0.2 \quad \pm 0.3\end{array}$ & $4.0 \pm 0.1$ & $452.36 \pm 0.07$ \\
\hline 2nd & $\begin{array}{r}85.7 \times 35.3 \\
\pm 0.3 \quad \pm 0.5\end{array}$ & $7.1 \pm 0.3$ & $241.85 \pm 0.04$ \\
\hline $3 r d$ & $\begin{array}{r}66.6 \times 30.0 \\
\pm 2.4 \quad \pm 1.3\end{array}$ & $6.5 \pm 0.5$ & $107.52 \pm 0.28$ \\
\hline
\end{tabular}

Table 3. Results of a point source fit in the $u v$ plane for the jet components.

\begin{tabular}{ccccc}
\hline Epoch & $\begin{array}{c}\text { Jet } \\
\text { Comp. }\end{array}$ & $\begin{array}{c}\Delta \alpha \cos \delta \\
(\mathrm{mas})\end{array}$ & $\begin{array}{c}\Delta \delta \\
(\mathrm{mas})\end{array}$ & $\begin{array}{c}\text { Flux Density } \\
\text { (mJy) }\end{array}$ \\
\hline \multirow{2}{*}{ 2nd } & North & $+7 \pm 4$ & $435 \pm 4$ & $1.70 \pm 0.03$ \\
& South & - & - & - \\
\multirow{3}{*}{ 3rd } & North & $+7 \pm 3$ & $+611 \pm 20$ & $1.45 \pm 0.02$ \\
& South & $-31 \pm 7$ & $-460 \pm 10$ & $0.89 \pm 0.02$ \\
\hline
\end{tabular}

is given in Tables 2 and 3 . In all fits, the core position was kept fixed at the phase origin since we are dealing with self calibrated data. It is important to mention that model fitting in the $u v$ plane did not differ significantly from fitting in the image plane using the alternative AIPS task IMFIT. For a bright radio source, such as Cygnus X-3, the UVFIT results are nevertheless preferred since they avoid the intermediate step of CLEAN deconvolution.
Table 4. Physical parameters of the Cygnus X-3 core derived assuming equipartition.

\begin{tabular}{ccccc}
\hline Epoch & $\begin{array}{c}\text { Radio } \\
\text { Luminosity } \\
\left(\mathrm{erg} \mathrm{s}^{-1}\right)\end{array}$ & $\begin{array}{c}\text { Brightness } \\
\text { Temperature } \\
(\mathrm{K})\end{array}$ & $\begin{array}{c}\text { Minimum } \\
\text { Energy } \\
(\mathrm{erg})\end{array}$ & $\begin{array}{c}\text { Magnetic } \\
\text { Field } \\
(\mathrm{G})\end{array}$ \\
\hline 1st & $2.1 \times 10^{33}$ & $5.1 \times 10^{6}$ & $3.6 \times 10^{43}$ & $2.0 \times 10^{-2}$ \\
2nd & $1.1 \times 10^{33}$ & $6.0 \times 10^{6}$ & $1.5 \times 10^{43}$ & $2.3 \times 10^{-2}$ \\
3rd & $0.5 \times 10^{33}$ & $4.0 \times 10^{6}$ & $0.7 \times 10^{43}$ & $2.2 \times 10^{-2}$ \\
\hline
\end{tabular}

If equipartition of the energy between the relativistic particles and the magnetic field is assumed, we can derive some of the physical parameters in a radio source. We have carried out these calculations for central core of Cygnus X-3 based on the observed values in Table 2. The formulation by Pacholczyk (1970) has been used, together with a synchrotron optically thin spectral index of -0.6 (between $0.1-100 \mathrm{GHz}$ ) and a $10 \mathrm{kpc}$ distance. The corresponding results for the radio luminosity, brightness temperature, minimum energy content and magnetic field are given in Table 4. It is remarkable that the magnetic field estimates are practically the same in all three epochs in spite of the variations in radio luminosity.

\section{Determination of the ejection parameters}

The asymmetry in both brightness and position in the microquasar ejecta of Figs. 5 and 6 is normally interpreted in the context of special relativity effects. The jet component brighter and more distant from the core is the one approaching towards the observer, while the fainter and closer one is receding away from him/her. Figure 6 is also very reminiscent of the main figure in Mirabel \& Rodríguez (1994) showing relativistic expansion in the superluminal microquasar GRS $1915+105$, a source where such effects are stronger than in Cygnus X-3. The special relativity formulae as given by Mirabel \& Rodríguez (1999) will be used throughout this discussion for quantitative estimates of the ejection parameters. The proper motion of the approaching $\left(\mu_{a}\right.$, sign -$)$ and receding jet $\left(\mu_{r}, \operatorname{sign}+\right)$ is then given by:

$\mu_{\mathrm{a}, \mathrm{r}}=\frac{v \sin \theta}{D(1 \mp \beta \cos \theta)}$,

where $v$ is the true jet velocity, $\theta$ the inclination angle with the line of sight, $\beta=v / c$ and $D$ the distance to the source.

In our case, the proper motion of the ejecta can be inferred from the third epoch map by using the offsets $\Delta \alpha \cos \delta$ and $\Delta \delta$ listed in Table 3 and assuming an age of $66.0 \mathrm{~d}$ since the ejection. This age is assumed because it seems natural that the most external ejecta should be also the oldest. For the brighter northern approaching component, the proper motion is $\mu_{\mathrm{a}}=9.3 \pm 0.3$ mas day $^{-1}$. Similarly, for the fainter southern receding component we find $\mu_{\mathrm{r}}=7.0 \pm 0.2$ mas day $^{-1}$. A more direct and independent estimate of $\mu_{\mathrm{a}}$ can be also obtained from the 


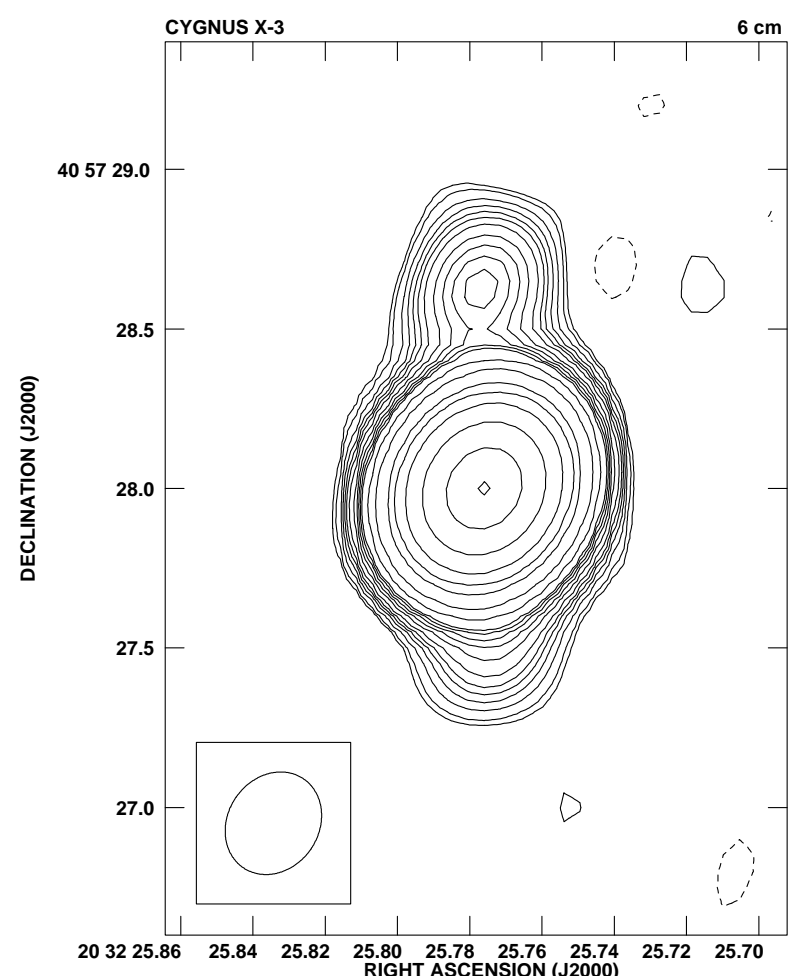

Fig. 5. Self-calibrated VLA map of Cygnus X-3 at the $6 \mathrm{~cm}$ wavelength obtained on 19/20 November 2000. Only visibilities in the interval not affected by variability have been used. The bipolar radio jets of Cygnus X-3 extending over one arcsecond are clearly developed at the third epoch of observation. Contours are $-3,3,4,6,8,10,12,15,20,25,30,35,40,60,100$, 200, 300, 500, 1000, 2000 and 3000 times $0.034 \mathrm{mJy} \mathrm{beam}^{-1}$, the rms noise. The ellipse at the bottom left corner represents the synthesized beam of $337 \times 285$ mas $^{2}$, with position angle of $-34^{\circ}$.

observed shift in the position of the northern jet component between the second and third epochs. Using the data in Table 3, the shift amounted to $176 \pm 24$ mas in a time interval of 15.05 days between the two epochs. The derived proper motion is therefore $\mu_{\mathrm{a}}=12 \pm 2$ mas day $^{-1}$. An extrapolation of this independent proper motion backward in time gives JD $2451817 \pm 7$ as the ejection date of the northern component. This epoch is close to one of the strongest outburst in the series of events that activated our ToO proposal, but differs in two weeks from the assumed date of creation for the oldest and most external ejecta (JD 2451802.5). Considering the important uncertainties in this extrapolation, we prefer to keep this assumption as a very reasonable initial epoch for proper motion estimates.

Having obtained values for the proper motion of both the jet and the counterjet, the ejection parameter $\beta \cos \theta$ can be easily obtained from Eq. (1):

$\beta \cos \theta=\frac{\mu_{\mathrm{a}}-\mu_{\mathrm{r}}}{\mu_{\mathrm{a}}+\mu_{\mathrm{r}}}$

It is remarkable that $\beta \cos \theta$ can be found without any knowledge of the distance $D$. Using the more accurate

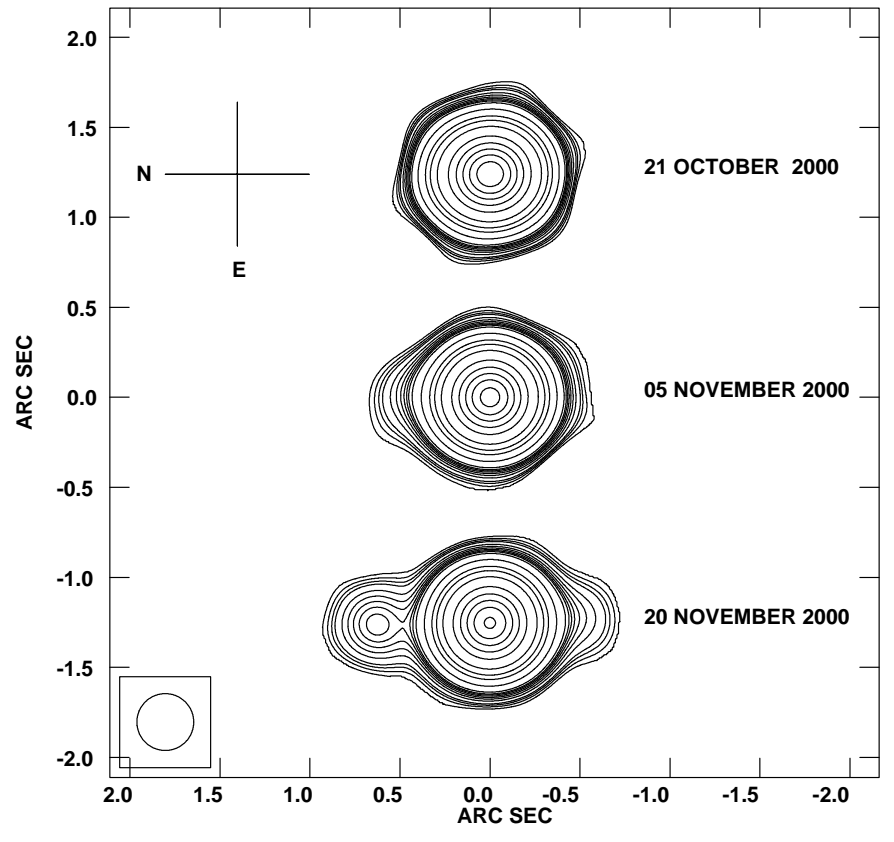

Fig. 6. Sequence of development of arcsecond radio jets in Cygnus X-3. The vertical offset between the different maps is proportional to the elapsed time. The maps have been rotated $90^{\circ}$ counterclockwise for easier display. In all maps, the clean components have been restored using an averaged circular beam of 361 mas diameter shown at the bottom left corner. Contours are 4, 6, 8, 10, 15, 20, 25, 30, 35, 40, 50, 100, 200, $300,500,1000,1500,2000,2500,3000$ times the rms noise of the map. The rms noise is $0.12,0.072$ and $0.034 \mathrm{mJy}^{b^{2}} \mathrm{bam}^{-1}$ for the top, middle and bottom panel, respectively.

proper motion values derived from the third epoch $(9.3 \pm$ 0.3 and $7.0 \pm 0.2$ mas day $\left.^{-1}\right)$, we obtain $\beta \cos \theta=0.14 \pm$ 0.03 . This implies a true jet velocity $v \geq(0.14 \pm 0.03) c$ and an ejection angle $\theta \leq 82 \pm 2^{\circ}$. We can also provide a relativistic upper limit for the Cygnus X-3 distance $D$ given by

$D \leq \frac{c}{\sqrt{\mu_{\mathrm{a}} \mu_{\mathrm{r}}}}$.

The resulting value $D \leq 21 \pm 1 \mathrm{kpc}$ is, however, not very constraining when compared to modern distance estimates to Cygnus X-3. For example, based on X-ray data Predehl et al. (2000) recently obtained a distance value of $9 \mathrm{kpc}$.

The inferred proper motions imply apparent subluminal velocities of $(0.54 \pm 0.02) c[D / 10 \mathrm{kpc}]$ and $(0.40 \pm$ $0.01) c[D / 10 \mathrm{kpc}]$ for the northern and southern jet components, respectively. Our direct (not-inferred) proper motion measurement for the northern jet component translates into a consistent apparent velocity of $(0.69 \pm$ $0.12) c[D / 10 \mathrm{kpc}]$. Accepting that the distance value to Cygnus $\mathrm{X}-3$ is $D=10 \mathrm{kpc}$, it is possible to derive both the true jet velocity and the inclination angle with the line of sight instead of the limits given above. Solving from Eq. (1) we get $\beta \sin \theta=0.46 \pm 0.03$, which implies $v=(0.48 \pm 0.04) c$ and $\theta=73 \pm 4^{\circ}$.

The flux density ratio between the approaching and receding jet components, $R \equiv S_{\mathrm{a}} / S_{\mathrm{r}}$, is related to the 
ejection parameters according to the Doppler boosting formula:

$R=\left(\frac{1+\beta \cos \theta}{1-\beta \cos \theta}\right)^{k-\alpha}$,

where $\alpha$ is the spectral index $\left(S_{\nu} \propto \nu^{\alpha}\right)$ and $k=2,3$ for a continuous jet or a discrete jet condensation. This formula is strictly valid for equal angular distances from the core of the approaching and receding components. This fact is relevant in our case because of the noticeable jet asymmetry. Therefore, the flux densities whose ratio is considered are not simultaneous in time according to the observer. By interpolation between the values in Table 3, the flux density of the northern component at 460 mas from the core is $1.66 \pm 0.03 \mathrm{mJy}$. This angular distance is the one at which we detect the southern component in the third epoch. Consequently, the flux density ratio is $R=1.87 \pm 0.08$. The Mioduszewski et al. (2001) maps suggest a continuous jet flow, so we will adopt $k=2$. Moreover, it seems natural to assume that the long-lived ejecta of Cygnus X-3 emits optically thin synchrotron radiation with a typical spectral index $\alpha=-0.6$. Under such circumstances and using Eq. (4), the $\beta \cos \theta$ parameter can be expressed as:

$\beta \cos \theta=\frac{R^{1 /(k-\alpha)}-1}{R^{1 /(k-\alpha)}+1}$,

that gives $\beta \cos \theta=0.12 \pm 0.01$. The error quoted does not include any uncertainty in the assumed spectral index. The resulting value is in excellent agreement with the independent estimate $\beta \cos \theta=0.14 \pm 0.03$ based on proper motion measurements.

\section{Discussion}

The VLA maps in this paper clearly indicate that relativistic expansion in Cygnus X-3 creates a two-sided radio jet extending over $\sim 1^{\prime \prime}$ or $\sim 0.05 \mathrm{pc}$ at the distance of this microquasar. Such a bipolar structure is completely different from the one-sided radio jet interpretation based on the VLBA maps by Mioduszewski et al. (2001). These authors imaged Cygnus X-3 several times with an angular resolution of a few mas in the days following the 1997 outburst event. The VLBA structures are interpreted by them as a curved one-sided radio jet, with strong Doppler boosting effect, extending over $\sim 100$ mas or $\sim 10^{-3}$ pc. Their estimated jet velocity is $\geq 0.81 \mathrm{c}$ based on the nondetection of the counterjet. The inclination angle with the line of sight of the approaching jet is consequently limited to $\leq 36^{\circ}$, a value further constrained to $\leq 14^{\circ}$ by their precessing jet models. In contrast, our observations point to radio jets being ejected with a large inclination angle and a velocity roughly half the speed of light. Incidentally, the velocity that we infer is similar to that recently reported for the lobes of the Sco X-1 (Fomalont et al. 2001). At this point, the obvious question is: how the VLA and VLBA structures can be reconciled? The scope of this problem is not limited to Cygnus X-3 only, as it may seem. In fact, some other microquasars occasionally appear as one-sided or two-sided when observed with the VLBA or the VLA. The superluminal systems GRS 1915+105 (Mirabel \& Rodríguez 1994; Dhawan et al. 2000) and GRO J1655-40 (Hjellming \& Rupen 1995; Tingay et al. 1995) are good examples of this statement.

\subsection{A one-sided versus a two-sided jet}

The Mioduszewski et al. (2001) results proposed a major change in our picture of the Cygnus X-3 ejecta. The previous two decades of interferometric observations suggested symmetric double or triple radio source structures, always with moderate relativistic velocities $(\sim 0.3 c)$. The works by Geldzahler et al. (1983), Spencer et al. (1986) and Schalinski et al. (1995) are good examples of such a statement. It is true that these historical interpretations were sometimes based on model fitting analysis using a limited number of baselines in the array. However, evidence for nearly symmetrical arcsecond structures around Cygnus X-3 is also present in good synthesis maps by independent observers such as Strom et al. (1989) and Martí et al. (2000). On the other hand, all observations by different authors are consistent with any radio jet structure in Cygnus X-3 (either one or two-sided) always being elongated approximately in the North-South direction. This fact immediately suggests that any precession of the jets is not of wide angle. Considering all the observed facts, the apparent contradiction may be due to several reasons:

1. The one-sided jet appearance at VLBA angular scales could be due to the over-resolving power of the array;

2. The ejection and physical jet parameters may vary substantially from outburst to outburst. However, a strongly precessing jet does not seem likely as mentioned above. On the other hand, the two sided arcsecond radio jet reported by Martí et al. (2000) was seen in the months following the same 1997 event observed by Mioduszewski et al. (2001);

3. It could also be possible that the identification by Mioduszewski et al. (2001) of the Cygnus X-3 core is not correct and, consequently, the jet is not actually one-sided. The objections against this possibility are that the proposed core remained stationary in position within 3 mas and that its VLBA flux density behaved just like the total of flux the source;

4. The motion of the ejecta could not be perfectly ballistic and deceleration may take place as the jets travel away and interact with the surrounding medium. If strong deceleration occurs, the one-sided jet appearance very close to the core, as seen with the VLBA, could be significantly different from what the VLA sees at significantly larger scales. The interaction of microquasar ejecta with the environment has been already observed in the case of XTE J1748-288, where jets were seen to collide with external material and decelerate (Hjellming et al. 1998); 
5. Alternatively, we could be dealing with jet obscuration by an absorbing medium instead of a Doppler boosting effect. This possibility was already tentatively suggested by Fender et al. (1999) for Cygnus X-3 and discarded by Mioduszewski et al. (2001) when considering the problems of having opaque material at $15 \mathrm{GHz}$ out to a least $\sim 1000 \mathrm{AU}$ away from the core.

None of the different possibilities considered above can be strictly ruled out with the available information. However, among them we believe that the absorption hypothesis is probably the most plausible one provided that we have a non-spherical and highly flattened wind. This suggestion is based on the recent estimate of the wind mass loss from the Woft Rayet companion in Cygnus X-3, that appears to be much higher than suspected. An enhanced mass loss would naturally imply that absorption effects are relevant in this case contrary to previous expectations. It is relevant to mention here that the absence of counter-jet in some VLBA images of GRS $1915+195$, within few hundred AU of the core, has been also interpreted as possibly due to thermal free-free absorption (Dhawan et al. 2000).

\subsection{Is the northern milli-arcsecond radio jet absorbed by a flattened disc-like wind?}

The Cygnus X-3 observations with the Infrared Space Observatory (IS0) by Ogley et al. (2001) provide a wind mass loss of $\dot{M}_{\mathrm{w}} \sim 10^{-4} M_{\odot} \mathrm{yr}^{-1}$ assuming spherical symmetry and an outflow velocity at infinity of $v_{\infty} \sim$ $1500 \mathrm{~km} \mathrm{~s}^{-1}$. If the wind geometry is not spherically symmetric but flattened in the plane of the binary orbit, the corresponding mass loss is different and depends on the solid angle covered by the wind. The infrared spectroscopic observations of Fender et al. (1999) clearly show a double peaked emission line of HeI with day to day variability. This fact strongly supports a significantly flattened disc-like wind, common for Wolf-Rayet stars, that is aligned with the orbital plane and extends far beyond the orbital size of a few solar radii. The inclination angle of the disk with the line of sight is unknown, but probably values of a few tens of degrees could be consistent with the small optical depth to X-rays.

According to Ogley et al. (2001), when their ISO observations are interpreted in the context of such a disc-like wind model, the corresponding mass loss may be as high as $1 \times 10^{-3} M_{\odot} \mathrm{yr}^{-1}$ sterad $^{-1}$ for a solid angle $10 \%$ of $4 \pi$. The total mass loss is then $1.3 \times 10^{-3} M_{\odot} \mathrm{yr}^{-1}$. Such a high value exceeds current estimates of powerful winds in massive Of/WN stars by one order of magnitude (Lang et al. 2001). In order to be cautious, we will adopt an intermediate mass loss of $5 \times 10^{-4} M_{\odot} \mathrm{yr}^{-1}$ for qualitative discussion in a flattened wind scenario. With a $\dot{M}_{\text {w }}$ value of this order, the interpretation of absorption by the wind at $15 \mathrm{GHz}$ does not look so unreasonable. The situation would be somewhat analog to the obscured radio jet in the Seyfert 2 galaxy NGC 4258. In this extragalactic object, the brightness asymmetry between jet and counterjet is

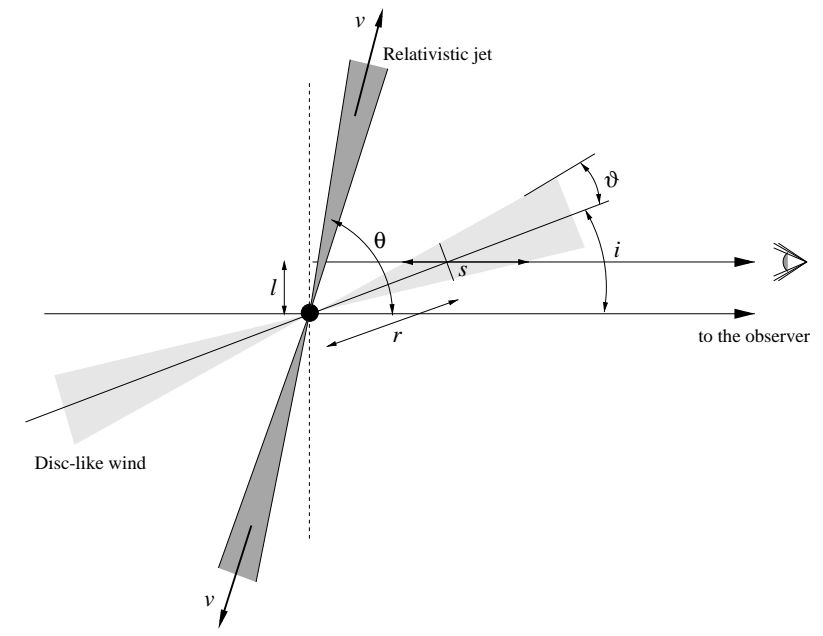

Fig. 7. Diagram showing the parameters involved in the obscuration interpretation of the apparent one-sided radio jet of Cygnus X-3 close to the binary system. The obscured jet is the one directed towards the observer, which later appears brighter than the counterjet when observed with the VLA due to relativistic aberration. In this interpretation, the jet flow velocity $v$ is not exactly perpendicular to the disc plane of the wind. However, the misalignment is a few tens of degrees at most.

related to thermal free-free absorption instead of Doppler boosting (Herrnstein et al. 1997).

In order to better check this possibility, it is instructive to estimate the optical depth of a line of sight through the wind disc. The geometric parameters involved are illustrated in Fig. 7. For simplicity, we will consider an unaccelerated, isothermal and fully ionized wind. A wind velocity of $v_{\infty}=10^{8} \mathrm{~cm} \mathrm{~s}^{-1}$, an electron temperature of $T_{\mathrm{e}}=10^{4} \mathrm{~K}$, a disc opening angle such that $\sin \vartheta=0.1$ and a disc inclination of $i=30^{\circ}$ will be also assumed as plausible values. The wind electron density $n_{\mathrm{e}}$ at a distance $r$ from the binary can be then expressed as:

$n_{\mathrm{e}}=\frac{\dot{M}_{\mathrm{w}}}{4 \pi r^{2} v_{\infty} m_{\mathrm{H}} \sin \vartheta}$,

where $m_{\mathrm{H}}$ is the proton rest mass. To be consistent with the VLBA images, the northern jet needs to be absorbed up to an angular distance of at least 50-100 mas. At $10 \mathrm{kpc}$, this corresponds to a linear size of $l=(0.75-1.5) \times$ $10^{16} \mathrm{~cm}(500-1000 \mathrm{AU})$ projected on the plane of the sky. A visual at this projected distance would cross the disc at a radius of $r=l / \sin i=(1.5-3.0) \times 10^{16} \mathrm{~cm}$, where electron densities of $(6.7-4.4) \times 10^{5} \mathrm{~cm}^{-3}$ are expected.

Assuming only free-free absorption by the ionized plasma, the absorption coefficient at radio wavelengths can be expressed as (Pacholczyk 1970):

$\kappa_{\nu}^{f f}=0.206 n_{\mathrm{e}}^{2} T_{\mathrm{e}}^{-1.38} \nu^{-2.08}$.

Based in Fig. 7, the path length of the line of sight through 
the disc can be approximated by $s \simeq 2 l \tan \vartheta / \sin ^{2} i=$ $(0.6-1.2) \times 10^{16} \mathrm{~cm}$. The corresponding optical depth is then:

$\tau_{\nu}^{f f} \simeq \kappa_{\nu}^{f f} s$,

where we approximate the absorption coefficient trough the line of sight by its value at the middle point in the path $s$ (see Fig. 7). From Eq. (8) at $\nu=15 \mathrm{GHz}$, we finally obtain $\tau^{f f} \simeq 1.15-0.14$ and the result would be even higher for lines of sight with smaller $l$. These calculations are not very sensitive to moderate variations in the parameters.

The proximity of the $\tau^{f f}$ values to unity makes difficult to completely reject that absorption may play a relevant role in the appearance of the Cygnus X-3 radio jets at the VLBA angular scales. Together with a flattened disc-like wind, the key of this interpretation is the recent and significantly higher wind mass loss estimates from ISO. Then, the wind would absorb the radio emission from the northern and brighter jet as suggested in Fig. 7. Although this interpretation is consistent with a large value of the jet angle with the line of sight, as derived above, the simple sketch in Fig. 7 suggests a relativistic jet flow not exactly perpendicular to the orbital and disc plane. Nevertheless, the perpendicularity could be still be preserved by assuming a warped disc with a geometry similar to the obscuring molecular disk in NGC 4258. In addition we could also have a narrow angle precession of the jet.

On the other hand, the proposed disc would be permanently emitting thermal free-free radio emission. It is then important to check that the expected level of thermal emission is consistent with the weakest flux densities ever observed for Cygnus X-3. Using the formulae of Panagia \& Felli (1975), we find that a thermal contribution of about $6 \mathrm{mJy}$ is expected at $15 \mathrm{GHz}$ for a spherical mass loss of $5 \times 10^{-4} M_{\odot} \mathrm{yr}^{-1}$. Of course our wind is not spherical, but we are only interested in an order of magnitude estimate. The thermal emission will be even lower at lower frequencies for the spectral index of a thermal wind $\left(S_{\nu} \propto \nu^{+0.6}\right)$. The expected emission is well consistent with the normal quiescent levels of Cygnus X-3 $(\leq 100 \mathrm{mJy}$ at $\mathrm{cm}$ wavelengths). Moreover, it is also very close to the quenched states of very low flux density $(\leq 5 \mathrm{mJy}$ at $15 \mathrm{GHz})$ prior to strong radio outbursts (Waltman et al. 1996).

\section{Conclusions}

The main results of this paper can be summarized as follows:

1. We have reported VLA radio observations that clearly reveal the development of bipolar, two-sided radio jets in the microquasar Cygnus X-3 in the weeks after a series of strong flaring episodes. Our VLA maps confirm the two-sided appearance of the radio jets when imaged at large distances from the binary system $(>0.01 \mathrm{pc})$.
2. The proper motions of both the jet and the counterjet have been estimated based on the most likely ejection date. For the northern jet component, our estimate is further consistent with a direct proper motion measurement between two consecutive epochs separated by two weeks. All this can be used to constrain the ejection parameters of the system. In particular, we obtain a value of $\beta \cos \theta=0.14 \pm 0.03$ that is in excellent agreement with an independent estimate based on the Doppler boosting effect. Assuming a $10 \mathrm{kpc}$ distance, we derive an ejection velocity of $(0.48 \pm 0.04) c$ with an angle of $73 \pm 4^{\circ}$ with the line of sight. No apparent superluminal velocities are observed.

3. Considering the new high values of the wind mass loss in Cygnus X-3 based on ISO data, as well as the likely disc-like structure of the wind, we tentatively suggest that the one-sided radio jet seen by Mioduszewski et al. (2001) is due to absorption effects in the vicinity of the binary system and not to a jet angle very close to the line of sight. If this is correct, the amount of Doppler boosting is better measured at the angular resolution of the VLA where absorption is negligible. Other interpretations discussed in the text are nevertheless not completely excluded.

Acknowledgements. We acknowledge partial support by DGICYT (PB97-0903), AYA2001-3092 and Junta de Andalucía (Spain). The National Radio Astronomy Observatory is a facility of the National Science Foundation operated under cooperative agreement by Associated Universities, Inc. The Green Bank Interferometer is a facility of the National Science Foundation operated by the NRAO in support of NASA High Energy Astrophysics programs. The authors also thank L. F. Rodríguez (UNAM, México) and an anonymous referee for helping to improve this paper, and G. G. Pooley (MRAO, UK) for making possible the on-line consultation of the Ryle Telescope monitorings.

\section{References}

Dhawan, V., Mirabel, I. F., \& Rodríguez, L. F. 2000, ApJ, 543,373

Fender, R. P., Hanson, M. M., \& Pooley, G. G. 1999, MNRAS, 308,473

Fomalont, E. B., Geldzahler, B. J., \& Bradshaw, C. F. 2001 [astro-ph/0104372]

Geldzahler, B. J., Johnston, K. J., Spencer, J. H., et al. 1983, ApJ, 273, L65

Giacconi, R., Gorenstein, P., Gursky, H., \& Waters, J. R. 1967, ApJ, 148, L119

Gregory, P. C., Kronberg, P. P., Seaquist, E. R., et al. 1972, Nat. Phys. Sci., 239, 114

Herrnstein, J. R., Moran, J. M., Greenhill, L. J., et al. 1997, ApJ, 475, L17

Hjellming, R. M., \& Johnston, K. J. 1988, ApJ, 328, 600

Hjellming, R. M., \& Rupen, M. P. 1995, Nature, 375, 464

Hjellming, R. M., Rupen, M. P., Mioduszewski, A. J., et al. 1998, AAS, $19310308 \mathrm{H}$ 
Lang, C. C., Goss, W. M., \& Rodríguez, L. F., ApJL, in press

Martí, J., Paredes, J. M., \& Estalella, R. 1992, A\&A, 258, 309

Martí, J., Paredes, J. M., \& Peracaula, M. 2000, ApJ, 545, 939

Mason, K. O., Cordova, F. A., \& White, N. E. 1986, ApJ, 309, 700

Mioduszewski, A. J., Rupen, M. P., Hjellming, R. M., Pooley, G. C., \& Waltman, E. B. 2001, ApJ, 553, 766

Mirabel, I. F., \& Rodríguez, L. F. 1994, Nature, 371, 46

Mirabel, I. F., \& Rodríguez, L. F. 1999, ARA\&A, 37, 409

Murthy, P. V. R., \& Wolfendale, A. W. 1993, in Gamma-Ray Astronomy (Cambridge University Press), 2nd edition, 197

Ogley, R. N., Bell Burnell, S. J., \& Fender, R. P. 2001, MNRAS, 322,177

Pacholczyk, A. G. 1970, Radio Astrophysics (Freeman, San Francisco)

Panagia, N., \& Felli, M. 1975, A\&A, 39, 1
Paredes, J. M., Martí, J., Ribó, M., \& Massi, M. 2000, Science, 288, 2340

Predehl, P., Burwitz, V., Paerels, F., \& Trümper, J. 2000, A\&A, 357, L25

Schalinski, C. J., Johnston, K. J., Witzel, A., et al. 1995, ApJ, 447,752

Spencer, R. E., Swinney, R. W., Johnston, K. J., \& Hjellming, R. M. 1986, ApJ, 309, 694

Strom, R. G., van Paradijs, J., \& van der Klis, M. 1989, Nature, 337,234

Tingay, S. J., Jauncey, D. L., Preston, R. A., et al. 1995, Nature, 374, 141

van Kerkwijk, M. H., Geballe, T. R., King, D. L., van der Klis, M., \& van Paradijs, J. 1996, A\&A, 314, 521

Waltman, E. B., Foster, R. S., Pooley, G. G., et al. 1996, AJ, 112,2690 